REVISTA PROYECCIONES $\mathrm{N}^{\circ}$ 9: 59-67

Julio 1985 - I.S.S.N. 0716-0917

\title{
UN TRATAMIENTO BAYESIANO ELEMENTAL PARA UN JUEGO EN FORMA NORMAL
}

Dr. ARNOLDO PRADO C. $\star$

RESUMEN.

Se presenta a través del ejemplo clásico del duopolio una metodología bayesiana para decidir en ambiente de incertidumbre sobre la conducta de los competidores. Se hace uso de un ejemplo elemental pre sentado por J. P. Aubin en el texto citado en la bibliografía. La cons trucción de las distribuciones a priori se suponen basadas en el histo rial de las relaciones recíprocas entre pares de competidores.

* Departamento de Matemáticas, Facultad de Ciencias, Universidad del Norte. 


\section{PRESENTACION DEL PROBLEMA.}

En el caso de un duopolio que enfrenta a dos productores de un cierto bien, bajo algunas consideraciones sencillas, la función de costo puede representarse en la forma

$F(x, y)=(x(x+y-u), y(x+y-u))$ con $x, y \in[0, u]=x=y$

Esta fórmula x e y representan las producciones del bien de cada uno de los productores; u es cierta constante que caracteriza la fini tud de los medios.

En términos de un juego $(\mathrm{X}, \mathrm{Y}, \mathrm{F})$ se encuentra rápidamente en un esquema no cooperativo que el punto de equilibrio de Cournot viene dado por:

$$
\bar{x}=\bar{y}=\frac{u}{3}
$$

Esta solución implica para los competidores un mismo costo:

$-\frac{u}{9}^{2}$

Ahora si ambos emplean el criterio de Stackelger se encuentra $\tilde{x}=\tilde{y}=\frac{2}{5}$ u lo que implica que ambos tienen un costo: $-\frac{2}{25} u^{2}$.

En el caso Cournot ambos competidores asumen el criterio del equilibrio no cooperativo, es decir si: $f(x, y)=x(x+y-u)$ es 1a función de costo de un productor $\mathrm{y} g(\mathrm{x}, \mathrm{y})=\mathrm{y}(\mathrm{x}+\mathrm{y}-\mathrm{u})$ la del otro, entonces $1 \mathrm{a}$ so lución de Cournot queda caracterizada por las siguientes relaciones simul táneas:

$$
\begin{aligned}
& f(\bar{x}, \bar{y})=\min _{x} f(x, \bar{y}) \\
& g(\bar{x}, \bar{y})=\min _{y} g(\bar{x}, y)
\end{aligned}
$$


Por el contrario, si el jugador B cuya función de costo es $f$ usa el criterio de Stackelberg entonces B aplica la estrategia $\tilde{x}$ tal que mini mice $f\left(x, y^{*}\right)$, donde $y^{*}$ es la respuesta estrategia escogida por su competi dor K, obtenida optimizando su propia función de costo g. Esto es $\mathrm{y}^{*}$ es el valor de $y$ que minimiza $g(x, y)=y(x+y-u)$. En el caso que nos interesa $y^{*}=\frac{1}{2}(u-x)$. Así en términos del productor $B$ :

$$
\begin{aligned}
& f\left(\tilde{x}, y^{*}\right)=\min _{x}\left(x, y^{*}\right) \\
& g\left(x, y^{*}\right)=\min _{y} g(x, y)
\end{aligned}
$$

Las distintas alternativas que se pueden presentar se resumen en el siguiente cuadro:

$$
f(x, x)=(f(x, y), g(x, y))
$$

\begin{tabular}{c|c|c}
\hline Cournot & Stackelberg \\
\hline Cournot & $\left(-\frac{1}{9} u^{2},-\frac{1}{9} u^{2}\right)$ & $\left(-\frac{1}{16} u^{2},-\frac{1}{8} u^{2}\right)$ \\
\hline Stackelberg & $\left(-\frac{1}{8} u^{2},-\frac{1}{16} u^{2}\right)$ & $\left(-\frac{2}{25} u^{2},-\frac{2}{25} u^{2}\right)$ \\
\hline
\end{tabular}

\section{Tabla 1}

En estas condiciones se puede replantear el problema introducien do la incertidumbre de cada jugador respecto a la regla de decisión que usa efectivamente su competidor.

Obviamente una primera aproximación al problema será plantear un nuevo juego entre $\mathrm{B}$ y $\mathrm{K}$, considerando ahora como espacios de estrategias $\{\mathrm{C}, \mathrm{S}\}$ para cada productor y como función de pérdida la matriz indicada en la tabla 1 . 
Readecuando la valores, el juego queda planteado en los siguien tes términos:

\begin{tabular}{c|cc}
\begin{tabular}{c|c}
$C$ & $C$
\end{tabular} & $S$ \\
\hline$C$ & $\left(-\frac{1}{9},-\frac{1}{9}\right)$ & $\left(-\frac{1}{16},-\frac{1}{8}\right.$ \\
\hline$S$ & $\left(-\frac{1}{8},-\frac{1}{16}\right)$ & $\left(-\frac{2}{25},-\frac{2}{25}\right.$ \\
\hline
\end{tabular}

Tabla 2

En esta situación, el principio de la racionalidad min-max condu ce al óptimo para B y para K si ambos escogen la estrategia C. Evidentemente lo más conveniente para ambas es la elección de S. Pero si esta elección la realiza, por ejemplo, sólo el competidor $B$, entonces, $\mathrm{K}$ se be neficia y B aumenta su costo.

\section{ENFOQUE BAYESIANO.}

Parece natural introducir en el modelo así planteado el análisis bayesiano que permite a cada productor proponer una distribución a priori sobre las estrategias de su competidor, para proceder en base de esa distribución a optimizar su propia función de costo.

$$
\text { Sea pues } \begin{aligned}
p & =\left(p_{s}, p_{c}\right)=\left(p_{1}, p_{2}\right) \\
q & =\left(q_{s}, q_{c}\right)=\left(q_{1}, q_{2}\right)
\end{aligned}
$$

Con $\mathrm{p}$ la distribución a priori que $B$ asigna al espacio (C,S) de $\mathrm{K}$ y $\mathrm{q}$ la distribución a priori que $\mathrm{K}$ asigna al espacio $(\mathrm{C}, \mathrm{S})$ de $\mathrm{B}$. EL significado de $p$ es que en opinión de $B, K$ escoge $C$ con probabilidad $p_{1}$ y $S$ con probabilidad $\mathrm{p}_{2}$. Análogamente para $\mathrm{K}$. 
En estas condiciones el costo esperado por $B$ con respecto a la situación conjunta $\left\{b_{i}, p_{j}\right\}$; donde $b=\left(b_{1}, b_{2}\right)$ es la distribución sobre su propio espacio $(C, S)$; será:

$$
\pi_{1}(b, p)=\sum_{i, j} p_{1}(i, j) b_{i} p_{j} \quad i, 1 \in\{c, s\}
$$

$\because=\left(P_{1}, q_{2}\right)$ es la función de costo conjunta de B,K definida en la tabla 2 .

Obviamente B escogerá b de modo de minimizar $\pi_{1}$.

$$
\begin{aligned}
& \pi_{1}(b, p)=-\frac{1}{9} b_{1} p_{1}-\frac{1}{16} b_{1} p_{2}-\frac{1}{8} b_{2} p_{1}-\frac{2}{25} b_{2} p_{2} \\
&=-\left(\frac{13}{3600} p_{1}-\frac{63}{3600}\right) b_{1}-\left(\frac{9}{200} p_{1}+\frac{16}{200}\right) \\
& 0 \leq b_{1} \leq 1 \Rightarrow \pi_{1}(b, p) \text { mínimo si } b_{1}=1 \text {. Este resultado indica }
\end{aligned}
$$

que el competidor $B$, en estado de incertidumbre sobre la conducta de $\mathrm{K}$, debe escoger siempre la estrategia C como lo sugiere el principio mini$\max$.

Asỉ el analista bayesiano dispone de reglas decimales racio nales totalmente definidas.

Análogamente $\mathrm{k}$ escogerá $\mathrm{k}=\left(\mathrm{k}_{1}, \mathrm{k}_{2}\right)$ de modo que la minimiza el costo esperado con respecto a la distribución $\left\{q_{i}, k_{j}\right\}$.

$$
\begin{aligned}
\pi_{2}(\mathrm{q}, \mathrm{k}) & =-\frac{1}{9} \mathrm{q}_{1} \mathrm{k}_{1}-\frac{1}{8} \mathrm{q}_{1} \mathrm{k}_{2}-\frac{1}{16} \mathrm{q}_{2} \mathrm{k}_{1}-\frac{2}{25} \mathrm{q}_{2} \mathrm{k}_{2} \\
& =-\left(\frac{13}{3600} \mathrm{q}_{1}-\frac{63}{3600}\right) \mathrm{k}_{1}-\left(\frac{9}{200} \mathrm{q}_{1}+\frac{16}{200}\right)
\end{aligned}
$$

$0 \leq k_{1} \leq 1 \Longrightarrow \dot{\pi}_{2}(q, k)$ mínimo si $k_{1}=1$. 


\section{ALTERNATIVAS DE GENERALIZACION.}

El análisis del duopolio sugiere varias generalizaciones inmedia tas. El caso más sencillo consiste en extender el número de competidores para llegar posteriormente a la situación general de $\mathrm{n}$ competidores en un mercado con m bienes. La primera generalización desde el punto de vista conceptual admite un análisis no cooperativo, y con una aproximación mas acorde con la realidad, un análisis cooperativo donde tengan cabida las eventuales coaliciones entre competidores, para llegar al planteamiento más complejo, en ambos contextos, cuando el número de bienes es m > 1 .

3.1. Caso de 1 bien con $n$ productores. Para $j=1,2, \ldots, n$, sean:

$x_{j}=$ espacio de producción del productor $j=[0, u]$, intervalo en $\mathbf{R}$.

$f_{j}=$ función de costo del productor $j$, en dominio $\prod_{j=1}^{n} x_{j} y$ valores reales.

$\left.E=t E_{I}, E_{2}\right\} \quad E_{1}=$ "criterio Cournot". $E_{2}=$ "criterio Stackelberg".

$\left.p_{i}=p_{i}^{j} / p_{i}^{j}=\operatorname{Prob} .\left\{E=E_{1}\right\}, j \neq i, j=1, \ldots, n\right\} ; p_{i}^{j}$ probabilidad a priori que i asigna a la elección de j del criterio Cournot.

$t_{i}=\left\{\left(t_{1}^{i}, t_{2}^{i}\right) / t_{1}=\operatorname{Prob} .\left(E=E_{1}\right), t_{1}+t_{2}=1\right\}$ : distribución en que i aleatoriza el espacio E.

Bajo estas circunstancias la decisión de $i$ viene dada por la dis tribución $\left\{t_{1}^{i}, t_{2}^{i}\right\}$ que minimiza con respecto a la distribución $\left(p_{i}, t_{i}\right)$, la expresión:

$E\left[f i\left(x_{1}, \ldots, x_{i-1}, x_{i}, x_{i+1}, \ldots, x_{n}\right)\right], \operatorname{con} x_{j}=\chi_{j}, \bar{x}_{j}$

$\bar{x}_{j}$ óptimo Cournot para $j$

$\stackrel{\sim}{\mathrm{x}}_{j}$ óptimo Stackelberg para $j$. 


\subsubsection{Estructura posible de $\mathbf{f}_{\mathbf{j}}$.}

Por extensión del duopolio, las funciones $\mathrm{f}_{j}$ admiten la forma

$$
\begin{aligned}
& f_{j}=x_{j}\left(\sum_{j=1}^{n}-u\right) \\
& \therefore \frac{\partial f_{j}}{\partial x_{j}}=0 \Longrightarrow \bar{x}_{j}=\frac{\sum_{l \neq j}^{\sum} x_{l}-u}{2} \ldots \\
& f_{j}\left(\bar{x}_{1}, \ldots, \bar{x}_{j-1}, x_{j}, x_{j+1}, \ldots, \bar{x}_{n}\right)=m_{j} f_{j}\left(\bar{x}_{1}, \ldots,\right.
\end{aligned}
$$

$\left.x_{j-1}, x \bar{x}_{j}+1, \ldots, \bar{x}_{n}\right)$ aun para $f_{j}$ así definido las estrategias Cournot $v$

Stackeberg quedan bien definidas.

\subsubsection{Expresión del valor esperado.}

El análisis del juego se supone realizado por un jugador i que prescinde de la hipótesis de un comportamiento bavesiano de sus competidores. Si introduce esta hipótesis entonces el análisis del jugador i debe incluir las distribuciones $p_{j}$ que cada jugador $j$ asigna a las carac teristicas $E_{1}$ y $E_{2}$ de sus competidores. Evidentemente el analista $i$ no puede conocer a priori estas distribuciones, sin embargo puede aceptar que ellas han sido generadas por la misma vỉa como él generó la suya. Por ejemplo, por el historial de las relaciones reciprocas entre pares de com petidores. Supuesto este hecho se puede plantear como hipótesis adicional que si $p_{i}(\dot{j})$ es la distribución que $i$ asigna a las elecciones que hará y sobre $\left\{E_{1}, E_{2}\right\}$ entonces $P_{i}(j)=P_{j}(i)$. Además, para los restantes competidores se debe cumplir la misma relación, esta es $p_{k}(\ell)=p_{i}(k)$. La consistencia de este argumento obliga a plantear como hipótesis adicio nal la siguiente relación con respecto al análisis que realiza el competí Jor i: 


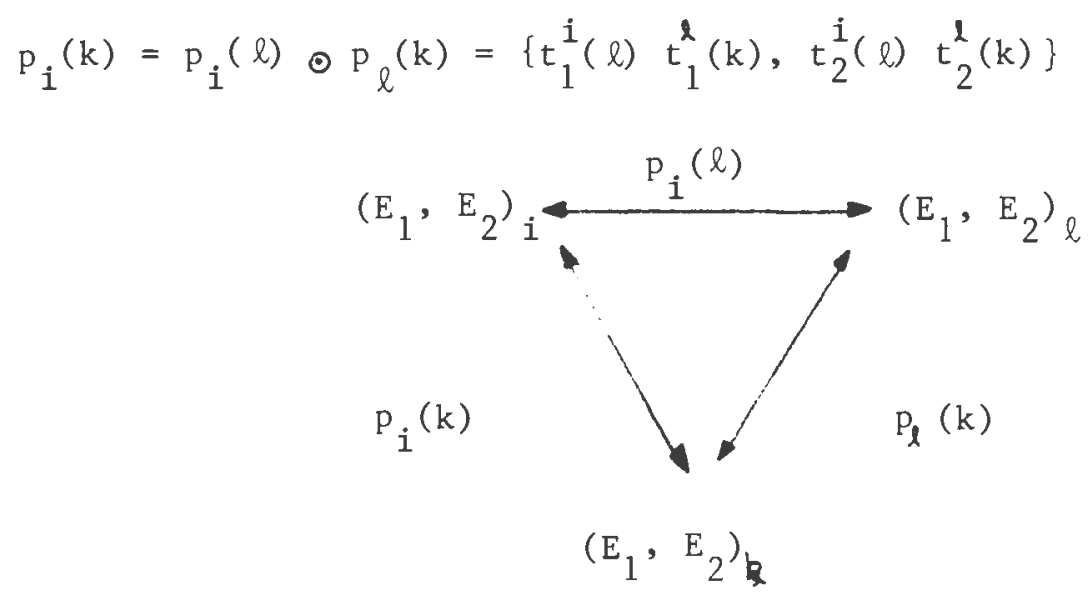

Bajo estos supuestos la decisión del analista se basa evidentemente sobre la distribución $t_{i}$ que minimice el valor esperado de la función $\mathrm{f}_{i}\left(\mathrm{x}_{1}^{*}, \ldots, \mathrm{x}_{i}, \ldots . \mathrm{x}_{n}^{*}\right)$ respecto a la distribución $t_{i}$, donde $\mathrm{x}_{j}^{\frac{1}{x}}, j \neq !$, es el valor de $\mathrm{x}_{j}$ óptimo para el jugador y obtenido minimizan do el valor esperado respecto de $t_{j}$ del valor esperado de la función $\mathrm{f}_{j}\left(\mathrm{x}_{1}, \ldots \mathrm{x}_{j}, \ldots \mathrm{x}_{\mathrm{n}}\right)$, respecto al conjunto de distribuciones $\mathrm{p}_{j}$ que en opinión del analista $i$, el competidor $j$ aplicaría al espacio $\left\{E_{1}, E_{2}\right\}$ de cada uno de sus n-1 competidores.

De acuerdo a su caracterización el conjunto de distribuciones $\mathrm{P}_{\mathrm{j}}$ asume la forma

$$
\begin{gathered}
p_{j}=\left\{p_{j}(l) / p_{i}(\ell)=p_{i}(j) \odot p_{j}(l), p_{i}(l)=\left(p_{i}^{l}, 1-p_{i}^{\ell}\right),\right. \\
\left.p_{i}(j)=\left(p_{i}^{j}, 1-p_{i}^{j}\right)\right\} .
\end{gathered}
$$

Se prueba fácilmente que si $\left(\mathrm{t}_{1}^{* j},{ }^{*} \mathrm{t}_{2}\right)$ es 1 a distribución óptima del competidor $j$, entonces existe un valor de $x_{j}\left(\bar{x}_{j} \circ \tilde{x}_{j}\right)$ que es equivalente en costo esperado con la distribución $\left(\begin{array}{c}t_{j}^{j} \\ 1\end{array},{ }^{* j}{ }_{2}\right)$. Este hecho origina, pues la existencia del valor $x_{j}^{*}$ óptimo para $j \neq i, j=1, \ldots, n$. 


\section{BIBL IOGRAF IA.}

AUBIN, J.P. "Mathematical Methods of Gane and Economic Theory" North-Holland. Noruega. 1982.

PRADO C., A Un juego bayesiano con valor de información.

Revista "Proyecciones", Departamento de Matemáticas, Universidad del Norte-Chile, Año 3, $\mathrm{N}^{\circ}$ 7, Junio 1984. 